\title{
Animal models of frailty: current applications in clinical research
}

This article was published in the following Dove Press journal:

Clinical Interventions in Aging

26 October 2016

Number of times this article has been viewed

\author{
Alice E Kane' \\ Sarah N Hilmer ${ }^{2-4}$ \\ John $\mathrm{Mach}^{2-4}$ \\ Sarah J Mitchell ${ }^{5}$ \\ Rafael de Cabo ${ }^{5}$ \\ Susan E Howlett' \\ 'Department of Pharmacology, \\ Dalhousie University, Halifax, NS, \\ Canada; ${ }^{2}$ Kolling Institute of Medical \\ Research and Sydney Medical School, \\ University of Sydney, ${ }^{3}$ Department of \\ Clinical Pharmacology, ${ }^{4}$ Department \\ of Aged Care, Royal North Shore \\ Hospital, Sydney, NSW, Australia; \\ ${ }^{5}$ National Institute on Aging, National \\ Institutes of Health, Baltimore, \\ MD, USA
}

\begin{abstract}
The ethical, logistical, and biological complications of working with an older population of people inherently limits clinical studies of frailty. The recent development of animal models of frailty, and tools for assessing frailty in animal models provides an invaluable opportunity for frailty research. This review summarizes currently published animal models of frailty including the interleukin-10 knock-out mouse, the mouse frailty phenotype assessment tool, and the mouse clinical frailty index. It discusses both current and potential roles of these models in research into mechanisms of frailty, interventions to prevent/delay frailty, and the effect of frailty on outcomes. Finally, this review discusses some of the challenges and opportunities of translating research findings from animals to humans.
\end{abstract}

Keywords: mouse models, frailty index, frailty phenotype, IL-10 knock-out

\section{Introduction}

Frailty is a state of high vulnerability for adverse health outcomes which reduces capacity to react to stressors. ${ }^{1}$ It is estimated that $25 \%-50 \%$ of those aged over 85 years are frail. ${ }^{1}$ Those who are frail have a significantly reduced quality of life, as well as being the largest users of health care services representing a large socioeconomic cost. $^{2}$ Clinical studies of frailty are inherently limited by the ethical, logistical, and biological complications of working with an older, more dependent, and heterogeneous population of people. There has been some clinical research into frailty, but there are still many unanswered questions. In particular, the mechanisms of frailty development, how, when, and which interventions may be effective, and the specifics of how frailty changes risks/outcomes are still not known. One of the reasons may be that until recently, there were no animal models of frailty. The recent development of several animal models of frailty is helping answer these questions.

This review briefly summarizes the importance and limitations of clinical frailty research, describes available animal models of frailty, and discusses both current and potential roles of these models in research into 1) mechanisms of frailty, 2) interventions to prevent/delay frailty, and 3) the effect of frailty on outcomes including surgical and medical treatments. Finally, this review discusses some of the challenges and opportunities of translating research findings from animals to humans.

\section{Frailty in humans}

\section{Frailty assessment in humans}

Clinically, frailty is measured and conceptualized in several different ways. It is generally considered a state of high vulnerability for adverse health outcomes and a reduced capacity to react to stressors. ${ }^{1}$ It can be assessed using a number of scales with varying 
degrees of difficulty, clinical applicability, and different underlying philosophies. ${ }^{3}$ Two of the most commonly used frailty assessment tools are the phenotype model and the frailty index (FI). The phenotype model was developed by Fried et $\mathrm{al}^{4}$ and conceptualizes frailty as a functional phenotype that categorizes someone as robust, pre-frail, or frail. This tool assesses frailty based on patients' poor functional performance in 5 criteria - weight loss, exhaustion, weakness, slow walking speed, and low physical activity. ${ }^{4}$ If patients have 3 or more of these criteria, they are considered frail; if they have 1 or 2 , they are considered pre-frail; and if they have none, they are considered robust. ${ }^{4}$ By contrast, the FI, as developed by Rockwood and colleagues, ${ }^{5}$ conceptualizes frailty as a multidimensional syndrome. It measures frailty by counting the number of health-related deficits accumulated by a person over his/her lifetime and focuses on the number of deficits rather than their nature. ${ }^{6,7}$ An FI score is calculated by summing the deficits a person displays across a variety of systems (eg, symptoms, laboratory results, diseases, and activities of daily living) and dividing by the maximum number of possible deficits. A higher FI score indicates a greater degree of frailty. ${ }^{7}$

\section{Clinical frailty research}

There is very little current understanding about the molecular basis of frailty, its development, and its mechanistic association with aging. The most common hypotheses about the pathophysiology of frailty are inflammatory cytokine dysregulation, ${ }^{8,9}$ mitochondrial dysregulation, ${ }^{10}$ and general immunosenescence, ${ }^{8}$ although it is likely that frailty involves a combination of physiological factors. ${ }^{11}$ There has been some recent clinical research into biomarkers of frailty, but the results have been conflicting. ${ }^{10}$ Despite a current focus on research into interventions to prevent, delay, or treat the onset of frailty, very little is known as the results of clinical studies have generally been inconclusive. ${ }^{12-16}$ Clinical studies of the effect of frailty on pharmacological and surgical outcomes, as well as outcomes following illness or other stressors, are limited. Pharmacological changes in frailty have not been extensively studied, and very little is known about drug dosing optimization or toxicity risk in frailty. ${ }^{17,18}$ Frailty is generally associated with poor outcomes following nonpharmacological interventions such as surgery, ${ }^{19}$ but there is little research into exactly how to manage and assess the risk of interventions in the frail population. Given the growing problem of frailty, it is important that we understand more about the mechanisms of frailty development, potential frailty interventions, and the effect of frailty on clinical outcomes.

\section{Challenges and limitations of clinical studies of frailty}

One of the main reasons for the lack of clinical studies on frailty is the inherent limitations of research in this population. Observational studies are limited by the heterogeneity of the older and frail populations, and randomized clinical trials are both logistically and ethically difficult. ${ }^{20}$ Nonetheless, the available information suggests that it is extremely important to consider, assess, and understand frailty in a clinical setting. Although clinical studies are beginning to understand frailty, there is still a real lack of evidence to guide clinicians in identifying, assessing, and dealing with frailty. ${ }^{11}$ Preclinical animal studies provide opportunities to provide this empirical evidence. In particular, animal studies can help us understand the mechanisms of frailty to identify potential biomarkers and explore interventions to modulate frailty. Furthermore, animal studies can help us understand changes in clinically relevant outcomes with frailty including responses to medical and surgical stressors.

\section{Animal models of frailty}

Animal models of aging have been used for decades to investigate both the underlying mechanisms of aging and interventions to increase lifespan and healthspan. ${ }^{21}$ The relatively short lifespans, reduced genetic diversity, control of environmental factors, and the ability to test a variety of outcomes including stress responses, metabolic outcomes, behavioral and functional assessments, and serum, urine, and tissue analysis make animal models invaluable tools in the study of aging. ${ }^{21}$ There has been a recent focus on developing animal models of frailty ${ }^{22}$ to explore mechanisms, interventions, and outcomes associated with frailty. These models have the same advantages as aging models. The currently available preclinical models of frailty are summarized in Table 1, and discussed as follows.

\section{Transgenic mouse models of frailty}

Interleukin (IL)-10 homozygous knock-out (KO) mice $\left(\mathrm{IL}-10^{\mathrm{tm} / \mathrm{tm}}\right)$ were developed as a model of colitis but were identified as showing a frail phenotype including inflammation and reduced muscle strength. ${ }^{9}$ The IL-10 KO mouse produces no IL-10, an anti-inflammatory cytokine, which results in a chronic inflammatory state via the increased expression of inflammatory mediators induced by nuclear factor-kappa B. ${ }^{23}$ Given this, Walston et al hypothesized that these transgenic mice may be used as a model of frailty. ${ }^{9}$ In a cohort of age-matched female C57BL/6 mice and IL- $10^{\mathrm{tm} / \mathrm{tm}}$ mice, they assessed mouse strength, activity, and weight 


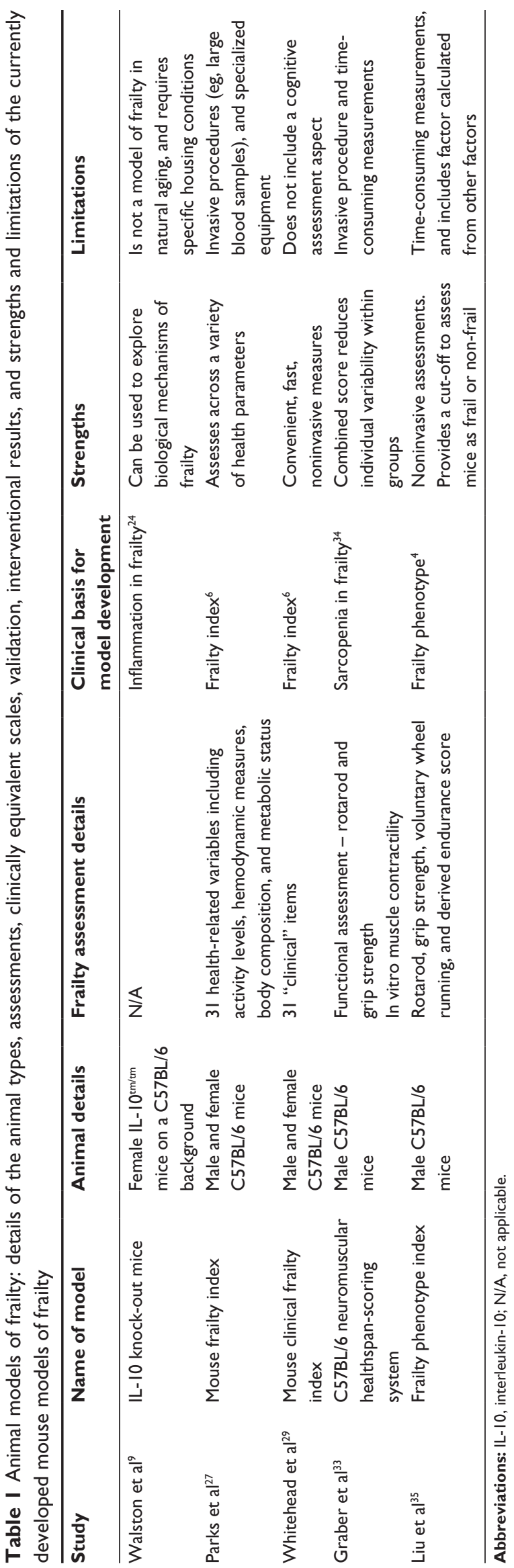

longitudinally, and measured serum IL-6 levels, and skeletal muscle gene expression at 8 and 50 weeks of age. ${ }^{9}$ They found that IL-10 KO mice had a faster decline in strength with age, and higher serum levels of the pro-inflammatory cytokine IL- 6 at 50 weeks of age, than wild-type mice. There were also changes in the IL-10 $1 \mathrm{~m}^{\mathrm{tm} / \mathrm{m}}$ mice in the skeletal muscle expression profiles of the genes related to apoptosis and mitochondria. ${ }^{9}$ These changes in strength and inflammatory serum markers are consistent with changes seen in frail humans. ${ }^{4,24}$ However, the mice do not appear to show all aspects of the frail phenotype as there was no difference between mouse groups in weight, activity level, or mortality up to 18 months. ${ }^{9}$

The characterization and validation of this mouse frailty model was continued by Ko et al. ${ }^{25}$ Male and female C57BL/6 and IL-1 $0^{\mathrm{tm} / \mathrm{tm}}$ mice were followed for up to 100 weeks, and mortality was investigated. Higher mortality was seen for both male and female IL-10 ${ }^{\mathrm{tm} / \mathrm{tm}}$ mice compared to controls, with log-rank analysis of Kaplan-Meier survival curves. They further investigated the inflammatory pathways and serum insulin-like growth factor-1 (IGF-1) levels in a cohort of these female mice. Serum cytokine levels were higher across female $\mathrm{KO}$ mice of almost all age groups compared to wild-types. Interestingly, the increase in IGF-1 was only seen in middle-aged mice, although the relationship between IGF-1 and age was significantly different across female IL-1 $10^{\mathrm{tm} / \mathrm{tm}}$ and wild-type mice. The authors concluded that these findings support the use of the IL-10 KO mouse as a model of frailty. ${ }^{25}$

There are limitations to using IL-10 $10^{\mathrm{tm} / \mathrm{mm}}$ mice as a model of frailty. These include the requirement for housing in pathogen-free barrier conditions to prevent the development of colitis, anemia, and early mortality. ${ }^{26}$ In addition, because this is a transgenic mouse model, the translatability of findings to the development of frailty in naturally aging humans is unclear. Furthermore, there are some key elements of the human phenotype assessment which are not observed or have not yet been investigated ${ }^{4}$ in these mice. These include no observed change in weight or activity level in the transgenic mice compared to controls, and a lack of investigation into endurance and walking speed. ${ }^{9}$

It has not yet been investigated, but it is possible that transgenic mouse models of premature aging such as the Werner syndrome mice or the Hutchinson-Gilford mice ${ }^{21}$ could also be used to investigate frailty. Biological changes such as inflammation and sarcopenia, as well as the mouse frailty phenotype and the mouse clinical FI could be easily assessed in premature aging models resulting in faster and 
cheaper studies than those that use naturally aging mice. However, the disadvantage of such models would be that it is unclear whether the mechanisms of frailty and aging development would be the same as those seen in natural aging.

\section{Mouse clinical FI}

Our group was the first to develop a frailty assessment tool for use in naturally aging mice. ${ }^{27}$ We developed a mouse FI assessment tool based on the deficit accumulation assessment tools used in humans. ${ }^{5,7}$ In a cohort of male and female C57BL/6 mice (adult, 12 months; old, 30 months), 31 health-related variables were identified and measured. These included activity measures such as movement velocity, hemodynamic measurements, body composition, and basic metabolism measurements. The results of these assessments were coded as deficits based on the number of standard deviations (SDs) from the mean reference values with more than 1 SD difference coded 0.25 , and more than 4 SD difference coded 1.0. The FI score is calculated by determining the number of observed deficits, divided by the total number of possible deficits. ${ }^{27}$ As with the human FI assessments, ${ }^{5}$ this tool allows the calculation of a continuous FI score from 0 to 1 , whereby a higher score indicates a greater degree of frailty. We found that FI scores were higher in the old group than the adult group, and that scores were similar in males and females. We also found that age-related cardiac changes including myocyte hypertrophy and lower peak contractions were associated with increasing FI scores. The main limitation of this assessment tool is the use of time-consuming and specialized equipment, as well as invasive procedures like blood sampling. We did conduct some preliminary investigations into the use of an 8-item noninvasive FI including 7 activity measures plus mouse weight. We also found an age-dependent increase in FI scores assessed with this tool, and an association with cardiac myocyte hypertrophy. ${ }^{27}$ Nevertheless, the heterogeneity increased dramatically as the number of items in the FI decreased, supporting the need to use more than 30 items as in human work. ${ }^{28}$

Whitehead et $\mathrm{al}^{29}$ continued this work on the development of a mouse FI. We developed a mouse clinical FI which utilizes 31 simply assessed, and noninvasive, clinical measures to quantify frailty in $\mathrm{C} 57 \mathrm{BL} / 6 \mathrm{~J}$ mice. These items cover a range of physiological systems including the musculoskeletal system, the ocular system, and the digestive system. We found a graded increase in FI scores from adult (5 months), to old (19 months) and very old (28 months) male and female C57BL/6 mice. The validity of this scale was correlated against human FI data from the Survey of Health, Aging and Retirement in Europe and was found to have good agreement. ${ }^{29}$ Furthermore, the rate at which deficits accumulated in the mice was similar to that seen clinically. ${ }^{30}$ We also compared the clinical 31-item index with the 8-item performance-based index proposed by Parks et al. ${ }^{27}$ We found that FI scores calculated with the 8 -item index were not different between 9 and 28 months of age in mice, and that the 8-item FI did not correlate with the human FI data. Based upon this data, we concluded that the 31-item mouse clinical FI is a valuable tool for use in longitudinal and interventional mouse aging and frailty studies. The main limitation of this tool is that it does not assess all systems. For example, it does not include a cognitive assessment item. Furthermore, it has only been validated in C57BL/6 mice. We do suggest, however, that other health-related deficits could be easily added to the index, particularly for the assessment of other mouse strains or species. ${ }^{29}$

Two studies have investigated the inter-rater reliability of the mouse clinical FI. Feridooni et $\mathrm{al}^{31}$ compared FI assessments of two independent raters for 233 older male C57BL/6 mice (11.5-14 months). The mice were assessed in 3 groups with discussion and refinement of assessment descriptors between each group. We found that inter-rater reliability increased over the study period as techniques were discussed and refined, and that overall, there was high (intra-class correlation coefficient $[\mathrm{ICC}]=0.77$ ) inter-rater reliability. ${ }^{31}$ Kane et al assessed inter-rater reliability between raters for 3 mouse FI studies, and also found overall high inter-rater reliability for the mouse clinical FI. We identified, however, poor inter-rater reliability $(\mathrm{ICC}=0.201-0.489)$ between raters of different professional backgrounds but excellent inter-rater reliability ( $\mathrm{ICC}=0.88$ ) between raters of the same background. We identify that care should be taken in the selection of raters for this assessment. ${ }^{32}$ Taken together, these data suggest that the mouse FI is a reliable, convenient, and fast assessment tool that has great value as a pre-clinical model of frailty.

\section{Mouse frailty phenotype assessment}

Graber et $\mathrm{al}^{33}$ developed a neuromuscular healthspan-scoring system (NMHSS) in male C57BL/6 mice. It was based on the concept of sarcopenia development in old age and frailty, ${ }^{34}$ and the need for a composite assessment tool to assess the contribution of this to healthspan. The score is calculated from the summed scores of a mouse's performance in the rotarod and inverted-cling grip tests, plus an in vitro muscle contractility assessment. In 3 groups of male C57BL/6 mice (6-7 months, adult; 24-26 months, old; 28 months, elderly), an age-related decline was seen in each of the functional outcomes. Contractility of the extensor digitorum longus 
muscles, primarily assessed by peak tetanic force, also declined with age. Although the combined NMHSS score reduced the variability within age groups, which the authors identify as a significant advantage in evaluating the effect of interventions, there was no clear age effect on NMHSS, with a decrease in mean score seen in the old, but not elderly mouse groups. ${ }^{33}$ This may have been a result of survivor bias. Another limitation of this tool is the invasive and terminal nature of the muscle contractility assessment, limiting the use of this tool in longitudinal studies.

Based upon this work, Liu et $\mathrm{al}^{35}$ developed a mouse frailty assessment tool based on the human phenotype model of frailty. This assessment tool assesses a mouse as frail based on its grip strength (assessed with the inverted-cling grip test), maximal walking speed (assessed by rotarod), physical activity (assessed by voluntary wheel running), and endurance (composite score of rotarod and grip strength performance). As with the clinical phenotype assessment, a mouse is defined as non-frail, mildly frail, or frail according to its performance in these assessments. A cut-off value which is $1.5 \mathrm{SD}$ below the cohort mean is determined for each assessment. A mouse with 3 or more criteria below this cut-off is considered frail, 2 criteria below the cut-off is considered mildly frail, and less than 2 criteria is considered nonfrail. In a cohort of 11 (27-28 months) male C57BL/6 mice, 1 mouse was identified as frail, and 1 as mildly frail. The authors concluded that this prevalence $(9 \%)$ of frailty was similar to that seen in a community-dwelling population of humans of an equivalent age of 76-84 years, ${ }^{4}$ although other clinical studies identify much higher incidences of frailty for that age group. ${ }^{1,36}$ Other limitations include time-consuming assessments and the requirement for specialized equipment, potentially limiting its use in many laboratories, and in longitudinal studies. Furthermore, the criteria do not completely mimic the human phenotype assessment as there is no weight loss factor, and the endurance factor is calculated from 2 of the other measured outcomes. The authors do identify that other criterion could be used in this assessment, such as treadmill running to exhaustion as an endurance measure. ${ }^{35}$ The addition of a weight loss factor, and a non-derived endurance measure may improve the translatability of this preclinical model.

\section{Current and potential roles of animal models of frailty in frailty research Animal studies of mechanisms of frailty} Several studies have investigated mechanistic changes with frailty in a variety of preclinical animal models of frailty (Table 2). Firstly, Parks et al, ${ }^{27}$ in developing the mouse FI, also looked at the association of physiological changes in isolated cardiac myocytes with frailty. In aged (30 months) male and female C57BL/6 mice, we found that cellular changes including hypertrophy and reduced contraction size, which had been traditionally associated with aging, were actually more closely associated with frailty. ${ }^{27}$ Clinically, there is evidence that frailty is more common in those with cardiovascular disease than those without, and those who are frail and have cardiovascular disease have a worse prognosis than those with the disease who are non-frail. ${ }^{37}$ Thus, understanding the association between frailty and cardiac changes is extremely important, and more preclinical studies of mechanisms of cardiac changes in frailty would be of benefit.

Walston et al have completed several studies in the IL-10 KO mouse exploring mechanistic changes across several physiological systems. In their early studies validating the IL-10 KO mouse, they found apoptosis and mitochondrial gene changes in skeletal muscle of the $\mathrm{KO}$ mice compared to wild-type C57BL/6 mice. ${ }^{9}$ They also saw changes in serum inflammatory cytokine levels. ${ }^{25}$ Sikkaa et al ${ }^{38}$ investigated cardiovascular changes in old (9 months) IL-10 KO mice and found that $\mathrm{KO}$ mice had stiffer blood vessels, impaired vascular relaxation, larger hearts, and contractile dysfunction compared to wild-type mice. These changes confirm what was seen at a cellular level by Parks et $\mathrm{al}^{27}$ in a different preclinical frailty model. Akki et al (2014) used the IL-10 $\mathrm{KO}$ mouse model and ${ }^{31} \mathrm{P}$ magnetic resonance spectroscopy to explore changes in adenosine triphosphate (ATP) kinetics in skeletal muscle in vivo in frailty. They found lower rates of ATP synthesis and reduced energy release from ATP hydrolysis in old (23 months) male IL-10 KO mice compared to wild-types. The authors suggest that, although this shows that ATP energetic abnormalities occur in skeletal muscle in this frail mouse model, it does not prove that muscle weakness is caused by these changes. ${ }^{39} \mathrm{Ko}$ et al continued work on skeletal muscle changes in the IL-10 KO mice, using old (22-24 months) female mice. They found reduced mitochondrial death signaling, altered formation of autophagosomes, and higher levels of damaged mitochondria in skeletal muscle of IL-10 KO mice compared to wild-types. These results imply that there is impaired mitochondrial degradation in this mouse model of frailty. ${ }^{40}$ Overall, the studies in the IL-10 KO mouse model of frailty suggest interesting potentially mechanistic changes. However, it is difficult to conclude whether these changes are a result of frailty and/or chronic inflammation, or whether they are a direct result of the lack of IL-10 in the model. It would be interesting to complete similar studies across a range of physiological systems in mouse models of frailty in the context of natural aging. ${ }^{29,35}$ 


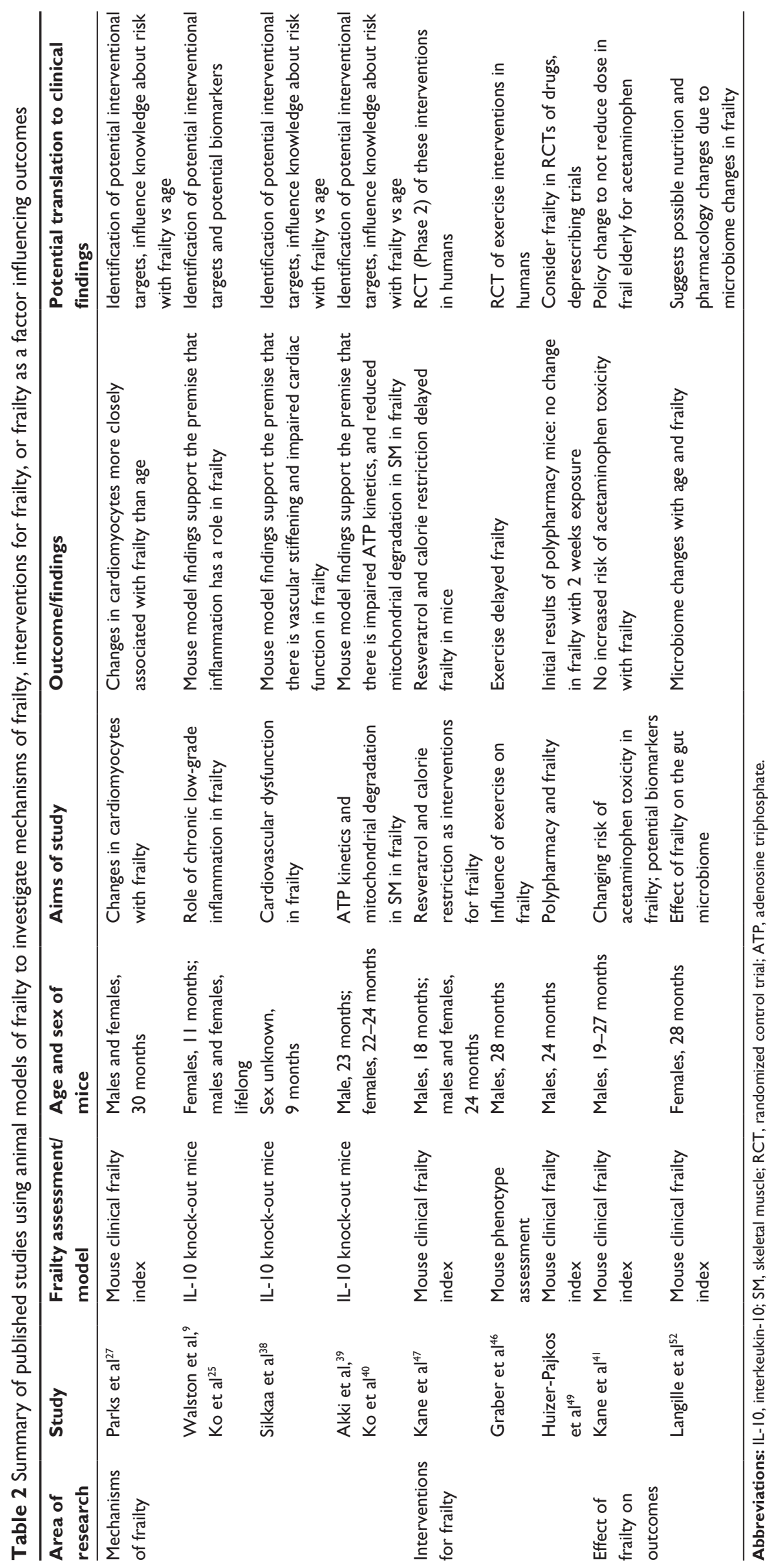


Kane et $\mathrm{al}^{41}$ looked at the association of frailty with potential biomarkers as a secondary outcome in a study of acetaminophen toxicity using the mouse clinical FI. There was a negative association between FI scores and serum albumin, protein, and alkaline phosphatase in old male C57BL/6 mice. Interestingly, similar observations have been reported in humans. ${ }^{42,43}$ However, these findings need to be confirmed in larger studies before conclusions can be made about the use of these as serum biomarkers of frailty. ${ }^{41}$

Although the mentioned studies have begun to look at physiological and potentially mechanistic changes with frailty, as well as biomarkers of frailty, there are many more studies that can and should be done. For example, changes in cardiovascular, inflammatory, and skeletal muscle systems with frailty could be further explored. These animal models of frailty, especially the tools that allow the quantification of frailty in aging models, provide researchers with the ability to look at the association of physiological changes across every system with frailty, to try and understand the underlying mechanisms of frailty. Furthermore, frailty can be included as a factor in longitudinal animal investigations of age-related changes and mechanisms, or geroscience investigations, to understand the mechanisms of development of both aging and frailty and their relationship.

\section{Animal studies of interventions to delay/ treat/prevent frailty}

Given the relatively new nature of tools that measure frailty in animals, studies of interventions are very limited (Table 2). A few studies have explored the effect of interventions on other outcomes associated with frailty such as sarcopenia ${ }^{44}$ or activity, ${ }^{45}$ but the focus of this review is on studies explicitly assessing frailty.

Using the mouse frailty phenotype assessment, Graber et al showed that 4 weeks of voluntary exercise reversed frailty in old (28 months) male C57BL/6 mice. ${ }^{46}$ Additionally, exercise improved the mean endurance and walking speeds of the intervention group compared to controls. ${ }^{46}$ Furthermore, they detected morphological changes in skeletal muscle from the exercise group compared to controls, including increased cross-sectional area and increased force. These observations demonstrated that even short-term voluntary exercise was able to improve functional aspects of the frailty phenotype, although the small frail sample size made the effect of the intervention on frailty difficult to determine. Further studies on exercise as a potential frailty intervention using both the phenotype model and mouse clinical FI would be of benefit.
Kane et a $\mathrm{a}^{47}$ used the mouse clinical FI to show that resveratrol treatment and calorie restriction delay frailty in mice. FIs were measured in old (18 months) male and female C57BL/6 mice fed either a calorie-restricted (CR) diet (40\% less calories than standard) or a standard diet. CR diet significantly reduced the FI score in male mice, with a trend observed for female mice. Another pro-longevity intervention, resveratrol $(100 \mathrm{mg} / \mathrm{kg})$, significantly lowered the FI score in mice fed the resveratrol diet compared to a control. ${ }^{47}$ Of particular importance is that FI was also investigated in DBA/2J mice, classically considered a short-lived strain, and we found that at the same biological age, the DBA/2J male mice had a higher FI than the C57BL/6 male mice. This was the first study to show that pharmaceutical and dietary interventions can delay frailty in mice. It not only provides preclinical evidence for exploring the effect of these interventions in humans but also shows the value of the mouse clinical FI as a preclinical tool for assessing possible interventions for frailty.

Many more frailty interventions could be explored in the mouse model. Interventions targeting potential physiological mechanisms of frailty such as cardiovascular or inflammatory changes could be investigated. Furthermore, the effect of drugs thought to improve healthspan or delay age-related diseases on frailty could be studied using these tools. It would be incredibly important and informative to incorporate frailty measures into rodent studies investigating lifespan and healthspan interventions, especially given the recent focus on the importance of standardized measures of healthspan. ${ }^{48}$

On the opposite side of this spectrum of studies, it is also possible to use these mouse models of frailty to explore the effect of potentially detrimental interventions on frailty in the context of aging. Huizer-Pajkos et $\mathrm{al}^{49}$ measured frailty, using the mouse clinical FI, as one of several geriatric outcomes in a study of polypharmacy in mice. Therapeutic doses of 5 commonly used drugs were administered in the food of old (24 months) male C57BL/6 mice for $2-4$ weeks, and functional outcomes and frailty were assessed at baseline, and post-intervention. Although no change was observed in FI over the short treatment time, there was a reduction in walking speed, grip strength, and activity in the mice administered drugs compared to control mice. ${ }^{49}$ There is great potential for further studies that use frailty as a clinically relevant geriatric outcome.

\section{Animal studies of frailty as a factor influencing outcomes}

Few studies have explored the effect of frailty as a factor influencing outcomes (Table 2). Kane et al ${ }^{41}$ did explore 
the effect of both age and frailty, assessed with the mouse clinical FI, on acetaminophen toxicity risk. Young (4-10 months) and old (19-27 months) male C57BL/6 mice were exposed to acute, subacute, or chronic acetaminophen treatment, and the degree of hepatotoxicity was assessed. We found that there was no difference with age or frailty in the degree of toxicity induced by any of the treatment regimens. ${ }^{41}$ This is an important finding as it provides preclinical evidence to suggest that it is safe to use the same dose of acetaminophen in frail older patients as in young patients. Pain in the older population is often undertreated, ${ }^{50,51}$ so evidence to optimize the efficacy and safety of this analgesic in this population could provide considerable benefit. Further studies should use the preclinical frailty models to explore the safety and efficacy of other drugs commonly used in the older frail population.

In other work, Langille et $\mathrm{a}^{52}$ investigated the effect of frailty and age on the microbiome of female C57BL/6 mice. They found a difference in the taxonomic and functional composition of the microbiome with both age and frailty. However, the small sample sizes did not allow separation of the effects of frailty and age. The authors do suggest, however, that changes in the microbiome with age and frailty may impact outcomes including vitamin availability, carbohydrate metabolism, and pharmacology of certain drugs. ${ }^{52}$ Further studies on changes in the microbiome with frailty would be of considerable interest.

These 2 studies represent an important start in considering frailty as a factor affecting outcomes. Indeed, preclinical models of frailty should be used in future studies to explore not just the safety and efficacy of drugs but also the effect of frailty on other clinical outcomes such as postsurgery and in response to other stressors (ie, chemotherapy). In particular, the effect of frailty on the toxicity and pharmacokinetics of drugs that are of high risk, commonly used in the older population, or known to have potential different age-related effects could be examined. Studies could also investigate the effect of frailty on prehabilitation, rehabilitation, exercise, dietary, and surgical interventions in mice to provide evidence to guide risk assessment and outcome optimization for all older frail patients.

\section{Challenges and opportunities of translating findings in animals to humans}

The development of preclinical models of frailty has provided an invaluable opportunity to researchers. The animal frailty studies summarized in this review have already provided exciting data on physiological mechanisms and changes in frailty, potential frailty biomarkers and interventions, and the effect of frailty on pharmacological outcomes. There is now an opportunity for further studies into each of these areas to fill important gaps in our frailty knowledge and provide preclinical evidence to guide decision making in humans. Information gained in animal studies on frailty interventions could lead to randomized control trials of these interventions in humans to delay/prevent frailty and its detrimental consequences. Animal studies of frailty mechanisms could lead to the development of biomarkers to improve the identification of frailty and pre-frailty in all older people. Furthermore, it could lead to the identification of new interventional targets. Studies of frailty as an influence on outcomes could provide the preclinical evidence needed to stratify clinical trial or observational study populations by frailty status to provide clear empirical evidence for drug or surgery outcomes with frailty. This may then guide the dosing of drugs in frailty, and the risk assessment of surgical interventions in frailty.

There are, however, some challenges in translating these findings from animals into humans. The applicability of the type of frailty assessment tool used, the animal model tested (including strain, sex, diet, and environment), and the interventions considered must all be given careful thought. As these are relatively new tools, there is the potential for further development and refinement. For example, the association of the mouse phenotype assessment and mouse clinical FI with mortality has not yet been determined. ${ }^{22}$ Studies comparing the different frailty assessment tools, or the tools with the IL-10 frail mouse model, have also not been completed. There is also a need for more longitudinal studies characterizing the development of frailty over the lifespan. Longitudinal studies could also explore the association of frailty with aging phenotypes, and other outcomes such as mortality or resilience. Additionally, the importance of clinically relevant outcomes is paramount. Seldeen et $\mathrm{al}^{22}$ suggest several different parameters that may be added to the mouse frailty assessments including measures of anxiety, memory, balance, and pain. The mouse clinical FI could be easily modified with the addition of items such as a cognitive assessment, or the replacement or refinement of items to suit either a different mouse strain or laboratory environment. The mouse phenotype could also include further items, especially a weight loss and endurance assessment, and the functional assessments included could be replaced with other assessments optimized in different research environments. Future studies exploring the modification and optimization of the frailty assessment tools would be of considerable benefit.

Furthermore, most of these studies have only been completed in C57BL/6 mice, and the majority used males 
only. C57BL/6 mice are the classical inbred mouse strain commonly used for aging research due to the wealth of phenotypic and genotypic information available. ${ }^{21}$ They are also a popular model because they develop similar agerelated changes as are seen in humans including reduced activity and vascular, bone, and inflammation changes. ${ }^{53-56}$ C57BL/6 mice live for approximately 26-30 months, and they are responsive to interventions that extend lifespan such as calorie restriction and pharmaceuticals. ${ }^{57,58}$ Thus, they provide a good model for the assessment of frailty in the context of natural aging. However, recent evidence suggests that the response to interventions may be strain- and sex specific, and this is an important point to consider moving forward. ${ }^{59,60}$ The use of different mouse strains and species would increase the potential applicability of these frailty assessment tools ${ }^{10}$ and provide further translatability of the findings to humans. The tools may need to be adapted for use in other strains and species. For example, one study assessing frailty in the DBA/2J mice with the mouse FI found that all mice of this strain were deaf by 18 months. ${ }^{47}$ Thus, this item in the index saturates at any early age, and should be replaced by a different item more applicable to this strain of mouse. ${ }^{61,62}$ The use of both sexes in future studies is also important, especially as clinically, there do seem to be changes in the development of frailty between males and females. ${ }^{63}$ Still, despite the possibility of further studies to continue to refine and optimize these tools, these preclinical models of frailty provide powerful new tools to advance our understanding of the underlying biology of frailty.

\section{Conclusion/summary}

Frailty is a significant social factor, especially in an aging population. Limited clinical studies show that those who are frail are at higher risk of a range of adverse outcomes, and may also respond differently to pharmacological and surgical interventions. ${ }^{1,18,19}$ Despite this, there is limited knowledge and understanding from clinical studies about frailty mechanisms and biomarkers, frailty interventions, and the effect of frailty on clinical outcomes. Animal models of frailty provide an invaluable opportunity to investigate and understand aspects of frailty which cannot be explored in human studies. The recent development of both the IL-10 KO transgenic mouse frailty $\operatorname{model}^{9}$ and the frailty assessment tools for use in natural aging ${ }^{29,35}$ has led to several novel studies exploring the mechanisms of frailty development, potential interventions to attenuate frailty, and the effect of frailty on outcomes. These studies represent the beginning of investigations in this area, from which the potential for further preclinical frailty studies is huge.

\section{Disclosure}

SJM and RdC are supported by the intramural research program of the National Institute on Aging, National Institutes of Health. The authors report no other conflicts of interest in this work.

\section{References}

1. Collard RM, Boter H, Schoevers RA, Oude Voshaar RC. Prevalence of frailty in community-dwelling older persons: a systematic review. $J$ Am Geriatr Soc. 2012;60(8):1487-1492.

2. Clegg A, Young J. Frailty and organization of health and social care. In: Rockwood K, Theou O, editors. Frailty in Aging: Biological, Clinical and Social Implications. Basel: Karger; 2015:161-173.

3. deVries NM, Staal JB, van Ravensberg CD, Hobbelen JS, Olde RikkertMG, Nijhuis-van der Sanden MW. Outcome instruments to measure frailty: a systematic review. Ageing Res Rev. 2011;10(1):104-114.

4. Fried LP, Tangen CM, Walston J, et al; Cardiovascular Health Study Collaborative Research Group. Frailty in older adults: evidence for a phenotype. J Gerontol A Biol Sci Med Sci. 2001;56(3):M146-M156.

5. Mitnitski AB, Mogilner AJ, Rockwood K. Accumulation of deficits as a proxy measure of aging. ScientificWorldJournal. 2001;1:323-336.

6. Mitnitski AB, Mogilner AJ, MacKnight C, Rockwood K. The mortality rate as a function of accumulated deficits in a frailty index. Mech Ageing Dev. 2002;123(11):1457-1460.

7. Rockwood K, Mitnitski A. Frailty in relation to the accumulation of deficits. J Gerontol A Biol Sci Med Sci. 2007;62(7):722-727.

8. Collerton J, Martin-Ruiz C, Davies K, et al. Frailty and the role of inflammation, immunosenescence and cellular ageing in the very old: cross-sectional findings from the Newcastle 85+ Study. Mech Ageing Dev. 2012; 133(6):456-466.

9. Walston J, Fedarko N, Yang H, et al. The physical and biological characterization of a frail mouse model. J Gerontol A Biol Sci Med Sci. 2008; 63(4):391-398.

10. Mohler MJ, Fain MJ, Wertheimer AM, Najafi B, Nikolich-Žugich J. The frailty syndrome: clinical measurements and basic underpinnings in humans and animals. Exp Gerontol. 2014;54:6-13.

11. Hubbard RE, Theou O. Frailty: enhancing the known knowns. Age Ageing. 2012;41(5):574-575.

12. Theou O, Stathokostas L, Roland KP, et al. The effectiveness of exercise interventions for the management of frailty: a systematic review. J Aging Res. 2011;2011:569194.

13. Cadore EL, Rodríguez-Mañas L, Sinclair A, Izquierdo M. Effects of different exercise interventions on risk of falls, gait ability, and balance in physically frail older adults: a systematic review. Rejuvenation Res. 2013; 16(2):105-114.

14. Tjia J, Velten SJ, Parsons C, Valluri S, Briesacher BA. Studies to reduce unnecessary medication use in frail older adults: a systematic review. Drugs Aging. 2013;30(5):285-307.

15. Giné-Garriga M, Roqué-Fíguls M, Coll-Planas L, Sitjà-Rabert M, Salvà A. Physical exercise interventions for improving performance-based measures of physical function in community-dwelling, frail older adults: a systematic review and meta-analysis. Arch Phys Med Rehabil. 2014;95(4):753.e3-769.e3.

16. Bibas L, Levi M, Bendayan M, Mullie L, Forman DE, Afilalo J. Therapeutic interventions for frail elderly patients: part I. Published randomised trials. Prog Cardiovasc Dis. 2014;57(2):134-143.

17. Johnston C, Hilmer SN, McLachlan AJ, Matthews ST, Carroll PR, Kirkpatrick CM. The impact of frailty on pharmacokinetics in older people: using gentamicin population pharmacokinetic modeling to investigate changes in renal drug clearance by glomerular filtration. Eur J Clin Pharmacol. 2014;70(5):549-555.

18. Hubbard RE, O'Mahony MS, Woodhouse KW. Medication prescribing in frail older people. Eur J Clin Pharmacol. 2013;69(3):319-326.

19. Partridge JS, Harari D, Dhesi JK. Frailty in the older surgical patient: a review. Age Ageing. 2012;41(2):142-147. 
20. Harris R, Dyson E. Recruitment of frail older people to research: lessons learnt through experience. $J$ Adv Nurs. 2001;36(5):643-651.

21. Mitchell SJ, Scheibye-Knudsen M, Longo DL, de Cabo R. Animal models of aging research: implications for human aging and age-related diseases. Annu Rev Anim Biosci. 2015;3:283-303.

22. Seldeen KL, Pang M, Troen BR. Mouse models of frailty: an emerging field. Curr Osteoporos Rep. 2015;13(5):280-286.

23. Rennick D, Davidson N, Berg D. Interleukin-10 gene knock-out mice: a model of chronic inflammation. Clin Immunol Immunopathol. 1995; 76(3 Pt 2):S174-S178.

24. Walston J, McBurnie MA, Newman A, et al; Cardiovascular Health Study. Frailty and activation of the inflammation and coagulation systems with and without clinical comorbidities: results from the Cardiovascular Health Study. Arch Intern Med. 2002;162(20):2333-2341.

25. Ko F, Yu Q, Xue QL, et al. Inflammation and mortality in a frail mouse model. Age (Dodr). 2012;34(3):705-715.

26. Bristol IJ, Mahler M, Edward H. Interleukin-10 gene targeted mutation. JAXNotes. 1997. Available from: https://www.jax.org/news-and-insights/ 1997/october/il10-tm1 cgn-an-interleukin-10-gene-targeted-mutation. Accessed September 30, 2016.

27. Parks RJ, Fares E, Macdonald JK, et al. A procedure for creating a frailty index based on deficit accumulation in aging mice. $J$ Gerontol A Biol Sci Med Sci. 2012;67(3):217-227.

28. Ridda I, Lindley R, MacIntyre RC. The challenges of clinical trials in the exclusion zone: the case of the frail elderly. Australas J Ageing. 2008; 27(2):61-66.

29. Whitehead JC, Hildebrand BA, Sun M, et al. A clinical frailty index in aging mice: comparisons with frailty index data in humans. J Gerontol A Biol Sci Med Sci. 2014;69(6):621-632.

30. Mitnitski A, Song X, Skoog I, et al. Relative fitness and frailty of elderly men and women in developed countries and their relationship with mortality. J Am Geriatr Soc. 2005;53(12):2184-2189.

31. Feridooni HA, Sun MH, Rockwood K, Howlett SE. Reliability of a frailty index based on the clinical assessment of health deficits in male C57BL/6J mice. J Gerontol A Biol Sci Med Sci. 2015;70(6):686-693.

32. Kane AE, Hilmer SN, Huizer-Pajkos A, et al. Factors that impact on interrater reliability of the mouse clinical frailty index. J Gerontol A Biol Sci Med Sci. 2015;70(6):694-695.

33. Graber TG, Ferguson-Stegall L, Kim JH, Thompson LV. C57BL/6 neuromuscular healthspan scoring system. J Gerontol A Biol Sci Med Sci. 2013;68(11):1326-1336.

34. Janssen I, Heymsfield SB, Ross R. Low relative skeletal muscle mass (sarcopenia) in older persons is associated with functional impairment and physical disability. J Am Geriatr Soc. 2002;50(5):889-896.

35. Liu H, Graber TG, Ferguson-Stegall L, Thompson LV. Clinically relevant frailty index for mice. J Gerontol A Biol Sci Med Sci. 2013;69(12): 1485-1489.

36. Clegg A, Young J, Iliffe S, Rikkert MO, Rockwood K. Frailty in elderly people. Lancet. 2013;381(9868):752-762.

37. Singh M, Stewart R, White H. Importance of frailty in patients with cardiovascular disease. Eur Heart J. 2014;35(26):1726-1731.

38. Sikkaa G, Miller KL, Steppan J, et al. Interleukin 10 knockout frail mice develop cardiac and vascular dysfunction with increased age. Exp Gerontol. 2013;48(2):128-135.

39. Akki A, Yang H, Gupta A, et al. Skeletal muscle ATP kinetics are impaired in frail mice. Age (Dodr). 2014;36(1):21-30.

40. Ko F, Abadir P, Marx R, et al. Impaired mitochondrial degradation by autophagy in the skeletal muscle of the aged female interleukin 10 null mouse. Exp Gerontol. 2016;73:23-27.

41. Kane AE, Mitchell SJ, Mach J, et al. Acetaminophen hepatotoxicity in mice: effect of age, frailty and exposure type. Exp Gerontol. 2016;73: 95-106.

42. Schalk BW, Visser M, Deeg DJ, Bouter LM. Lower levels of serum albumin and total cholesterol and future decline in functional performance in older persons: the Longitudinal Aging Study Amsterdam. Age Ageing. 2004;33(3):266-272.
43. Kitamura K, Nakamura K, Nishiwaki T, Ueno K, Nakazawa A, Hasegawa M. Determination of whether the association between serum albumin and activities of daily living in frail elderly people is causal. Environ Health Prev Med. 2012;17(2):164-168.

44. Wang B, Yang G, Liang X, Zhu M, Du M. Grape seed extract prevents skeletal muscle wasting in interleukin 10 knockout mice. $B M C$ Complement Altern Med. 2014;14:162.

45. Lin CH, Yang H, Xue QL, et al. Losartan improves measures of activity, inflammation, and oxidative stress in older mice. Exp Gerontol. 2014; $58: 174-178$

46. Graber TG, Ferguson-Stegall L, Liu H, Thompson LV. Voluntary aerobic exercise reverses frailty in old mice. J Gerontol A Biol Sci Med Sci. 2015;70(9):1045-1058.

47. Kane AE, Hilmer SN, Boyer D, et al. Impact of longevity interventions on a validated mouse clinical frailty index. J Gerontol A Biol Sci Med Sci. 2016;71(3):333-339.

48. Richardson A, Fischer KE, Speakman JR, et al. Measures of healthspan as indices of aging in mice - a recommendation. J Gerontol A Biol Sci Med Sci. 2015;71(4):427-430.

49. Huizer-Pajkos A, Kane AE, Howlett SE, et al. Adverse geriatric outcomes secondary to polypharmacy in a mouse model: the influence of aging. J Gerontol Biol Sci Med Sci. 2016;71(5):571-577.

50. Horgas AL, Tsai PF. Analgesic drug prescription and use in cognitively impaired nursing home residents. Nurs Res. 1998;47(4):235-242.

51. Won AB, Lapane KL, Vallow S, Schein J, Morris JN, Lipsitz LA. Persistent nonmalignant pain and analgesic prescribing patterns in elderly nursing home residents. J Am Geriatr Soc. 2004;52(6):867-874.

52. Langille MGI, Meehan CJ, Koenig JE, et al. Microbial shifts in the aging mouse gut. Microbiome. 2014;2:50.

53. Ingram DK. Age-related decline in physical activity: generalization to nonhumans. Med Sci Sports Exerc. 2000;32(9):1623-1629.

54. Sindler AL, Fleenor BS, Calvert JW, et al. Nitrite supplementation reverses vascular endothelial dysfunction and large elastic artery stiffness with aging. Aging Cell. 2011;10(3):429-437.

55. Rowlatt C, Chesterman FC, Sheriff MU. Lifespan, age changes and tumour incidence in an ageing C57BL mouse colony. Lab Anim. 1976; 10(10):419-442.

56. Turturro A, Duffy P, Hass B, Kodell R, Hart R. Survival characteristics and age-adjusted disease incidences in $\mathrm{C} 57 \mathrm{BL} / 6$ mice fed a commonly used cereal-based diet modulated by dietary restriction. J Gerontol A Biol Sci Med Sci. 2002;57(11):B379-B389.

57. Goodrick CL. Life-span and the inheritance of longevity of inbred mice. J Gerontol. 1975;30(3):257-263.

58. Forster MJ, Morris P, Sohal RS. Genotype and age influence the effect of caloric intake on mortality in mice. FASEB J. 2003;17(6):690-692.

59. Liao CY, Rikke BA, Johnson TE, Diaz V, Nelson JF. Genetic variation in the murine lifespan response to dietary restriction: from life extension to life shortening. Aging Cell. 2010;9(1):92-95.

60. Mitchell SJ, Madrigal-Matute J, Scheibye-Knudsen M, et al. Effects of sex, strain, and energy intake on hallmarks of aging in mice. Cell Metab. 2016;23(6):1093-1112.

61. Searle SD, Mitnitski A, Gahbauer EA, Gill TM, Rockwood K. A standard procedure for creating a frailty index. BMC Geriatr. 2008;8:24.

62. Willott JF, Bross LS, McFadden S. Ameliorative effects of exposing DBA/2J mice to an augmented acoustic environment on histological changes in the cochlea and anteroventral cochlear nucleus. $J$ Assoc Res Otolaryngol. 2005;6(3):234-243.

63. Hubbard R. Sex differences in frailty. Interdiscip Top Gerontol Geriatr. 2015;41:41-53. 


\section{Publish your work in this journal}

Clinical Interventions in Aging is an international, peer-reviewed journal focusing on evidence-based reports on the value or lack thereof of treatments intended to prevent or delay the onset of maladaptive correlates of aging in human beings. This journal is indexed on PubMed Central, MedLine,
CAS, Scopus and the Elsevier Bibliographic databases. The manuscript management system is completely online and includes a very quick and fair peer-review system, which is all easy to use. Visit http://www.dovepress. $\mathrm{com} /$ testimonials.php to read real quotes from published authors. 